\title{
Cerebral abscesses produced by bacterial implantation and septic embolisation in primates
}

\author{
J A M E S H. W O O D, W I L L A M E. L I G H T F OOTE I I, A N D \\ A Y U B K . O M M A Y A \\ From the Surgical Neurology and Experimental Therapeutics Branches, National Institute of \\ Neurological and Communicative Disorders and Stroke, National Institutes of Health, \\ Bethesda, Maryland, USA
}

SUM MARY The degree of brain abscess encapsulation is positively related to surgical mortality and methods to enhance capsule wall formation, therefore, have therapeutic relevance. Two primate models are described which may be useful in the investigation of encapsulation of traumatic and metastatic brain abscesses. Direct intracerebral inoculation induces abscesses displaying more prominent inflammatory responses and encapsulation than does septic embolisation, despite similar abscess age and size. Cerebral ischaemia surrounding metastatic suppurative foci may retard capsule wall formation.

Approximately $70 \%$ of brain abscesses among civilian populations originate from either direct contamination during head trauma or distant systemic infection (Carey et al., 1972). Septic embolisation with abscess formation is not uncommon among cyanotic patients with right-to-left shunts secondary to congenital heart disease (Bhatia et al., 1976) or pulmonary arteriovenous malformations (Eberhard, 1969), or among patients who are deficient in immunoglobulins (Hoffman et al., 1970).

Although most bacterial infections of the central nervous system respond to antibiotic medications, the successful treatment of brain abscesses requires early surgical intervention. Computed tomography has recently replaced cerebral angiography in the diagnosis and postoperative evaluation of cerebral abscesses (New et al., 1976; Zimmerman et al., 1976, 1977; Joubert and Stephanov, 1977; Kaufman and Leeds, 1977). Despite these sophisticated neuroradiological procedures, the mortality of intracranial abscess remains high (Shaw and Russell, 1977), presumably because other factors thought to alter outcome (Le Beau et al., 1973; Shaw and

This paper was presented in part at the Annual Meeting of the American Association of Neurological Surgeons, Toronto, Canada, 25-28 April 1977.

Address for reprint requests: Dr James H. Wood, Division of Neurosurgery, University of Pennsylvania School of Medicine, 3400 Spruce Street, Philadelphia, Pennsylvania 19104, USA.

Accepted 4 July 1978
Russell, 1975; Van Alphen and Dreissen, 1976) and time of patient presentation (Shaw and Russell, 1977) have not been modified.

The overall mortality for cerebral abscesses varies from 20-50\% (Garfield, 1969; Carey et al., 1972; Le Beau et al., 1973; Bhatia et al., 1976; Val Alphen and Dreissen, 1976; Joubert and Stephanov, 1977; Shaw and Russell, 1977). At surgery, many brain abscesses present as poorly or non-encapsulated suppurative foci of cerebritis. The mortality of these acute abscesses is approximately twice that of chronic abscesses (Le Beau et al., 1973) which are usually encapsulated. Thus, the degree of encapsulation contributes significantly to the clinical outcome.

The purpose of this communication is to describe two primate models that may be useful in the study of traumatic and metastatic brain abscess encapsulation and to evaluate the pathological characteristics of cerebral abscesses experimentally produced by direct bacterial implantation and septic embolisation. The role of vascular proliferation on the angiographic appearance of experimental brain abscesses is discussed by Wood et al. (1978).

\section{Materials and methods}

Each of $214 \mathrm{~kg}$ adult rhesus monkeys (Macaca mulatta) was anaesthetised with incremental intravenous sodium thiamylal immediately before sur- 
gery. A small burrhole was placed in the right parietal bone of five animals; thereafter, $0.05 \mathrm{ml}$ of a $2 \%$ nutrient agar solution contaminated with pure cultures of Staphylococcus epidermidis (penicillin sensitive) was injected intracerebrally through the intact dura mater using a 30 gauge needle (Hassler and Forsgren, 1964). The agar had cooled to the point of early solidification at the time of injection so that the agar would not flow through the needle tract and contaminate the subarachnoid space. Three control animals underwent similar trephination procedures and direct intracerebral instillation of $0.05 \mathrm{ml}$ of sterile $2 \%$ nutrient agar. These intracerebrally inoculated monkeys then underwent scalp closure and received a single intramuscular dose of 600000 units of procaine penicillin $\mathrm{G}$ in oil.

Eight monkeys underwent right carotid arteriotomy and embolisation with $1.5 \mathrm{~mm} \times 8 \mathrm{~mm}$ cylinders (Microfil, Canton-Biochemical Products, Boulder, Colorado) using the technique described by Molinari (1972) and Laurent et al. (1975). These pliable cylinders were contaminated with pure cultures of Staphylococcus epidermidis (penicillin sensitive) before carotid arterial injection. Five control animals were embolised in a similar way with sterile silicone cylinders. These embolised animals received a single 600000 unit dose of intramuscular procaine penicillin $G$ in oil to prevent mycotic aneurysm formation and fatal subarachnoid haemorrhage (Molinari et al., 1973).

During the sixth week after operation the animals were anaesthetised with incremental intravenous sodium thiamylal and needle aspiration of the brain abscesses was attempted through right parietal burrholes. Specimens, when available, were cultured aerobically and anaerobically. The animals were then perfused with physiological saline followed with $10 \%$ buffered formalin. After removal, the brains were stored in $10 \%$ formalin for one week. Thereafter, the brains were cut into standard $4.5 \mathrm{~mm}$ thick coronal sec- tions. The brain sections were stained with haematoxylin and eosin, luxol fast blue-periodic acidSchiff and Masson's trichrome stains. Sections from each brain slice were stained simultaneously using techniques for minimising errors caused by tissue shrinkage.

Assuming the abscesses to be spherical, abscess volumes were estimated from diameter measurements. The presence of a significant mass effect was defined by a displacement of the midline structures greater than $2 \mathrm{~mm}$ from right to left.

\section{Results}

Intracerebral abscesses were produced in all five animals inoculated with septic nutrient agar. Cultures of the abscess aspirates from four of these monkeys grew Staphylococcus epidermidis (Table). Brain abscesses did not develop in the three animals inoculated with sterile agar, and attempts at aspiration were unproductive.

Six of the eight animals which underwent intracarotid embolisation with septic cylinders developed brain abscesses. Staphylococcus epider midis organisms were cultured from abscess aspirates from three of these monkeys. The abscess aspirate from one septically embolised animal was sterile, and attempts to aspirate fluid from the remaining two animals were unproductive. Fata massive ischaemic anterior paramedial infarction similar to those described by Laurent et al. (1975) resulted in the death of two animals within 24 hours of septic embolisation. The three monkeys which survived embolisation with sterile cylinders developed ischaemic infarctions without abscess formation. Massive fatal bland infarction of the anterior paramedian region occurred in one animal, and a massive fatal haemorrhagic infarction (Laurent et al., 1976) developed in another monkey within 24 hours of sterile embolisation. Attempts at needle aspiration in these animals were not productive.

Table Pathological data on intracerebral agar instillation and intracarotid embolisation

\begin{tabular}{|c|c|c|c|c|c|c|}
\hline $\begin{array}{l}\text { Surviving } \\
\text { monkeys }\end{array}$ & Procedure & $\begin{array}{l}\text { Aspirate } \\
\text { culture } \\
\text { S. epidermidis: } \\
\text { sterile }\end{array}$ & $\begin{array}{l}\text { Abscess } \\
\text { volume }+ \\
(m m \pm S E M)\end{array}$ & Encapsulation & $\begin{array}{l}\text { Inflammatory } \\
\text { response }\end{array}$ & $\begin{array}{l}\text { Displacement of } \\
\text { midline structures }\end{array}$ \\
\hline $\begin{array}{l}5 \\
3 \\
6 * \\
3 \dagger\end{array}$ & $\begin{array}{l}\text { Septic agar instillation } \\
\text { Sterile agar instillation (control) } \\
\text { Septic embolisation } \\
\text { Sterile embolisation (control) }\end{array}$ & $\begin{array}{l}4 / 5: 1 / 5 \\
3 / 6: 1 / 6\end{array}$ & $\begin{array}{l}961 \pm 121 \\
\text { None } \\
805 \pm 86 \\
\text { None }\end{array}$ & $\begin{array}{l}\text { Thick } \\
\text { None } \\
\text { Thin } \\
\text { None }\end{array}$ & $\begin{array}{l}\text { Intense } \\
\text { Minimal } \\
\text { Meagre } \\
\text { Minimal }\end{array}$ & $\begin{array}{l}5 / 5 \\
0 / 3 \\
0 / 6 \\
0 / 3\end{array}$ \\
\hline
\end{tabular}

*Two additional monkeys underwent fatal bland infarction within 24 hours of septic embolisation.

†One additional monkey underwent fatal haemorrhagic infarction and another animal underwent fatal bland infarction within 24 hours of sterile embolisation.

$¥$ Mean abscess volumes of the septically instilled and septically embolised animals were not significantly different (two-tailed Student’s $t$ test). SEM = standard error of mean; $S=$ Staphylococcus. 
Gross inspection of the abscess walls demonstrated profoundly thicker capsules among the animals which had received intracerebral injections of contaminated agar (Fig1A) than those among the septically embolised monkeys (Fig. 1B). The mean abscess volume estimates were $961 \pm 121$ $\mathrm{mm}^{3}( \pm$ SEM) in the five animals which received intracerebral contaminated agar inoculations and $805 \pm 86 \mathrm{~mm}^{3}$ in the six monkeys which underwent septic embolisation (Table). Assuming these abscesses to be spherical, the estimates of mean abscess volume were not significantly different (two-tailed Student's $t$ test). However, the midline structures were displaced more than $2 \mathrm{~mm}$ from right to left only in those animals which developed abscesses after instillation of contaminated agar (Fig. 1A). Areas of infarct cavitation were noted in brain tissue surrounding the abscesses in the animals which survived septic embolisation (Fig. 1B).

Light microscopic examination of the stained sections of abscesses developing after intracerebral instillation of septic agar revealed four zones: a central necrotic core, a thick medial layer of acute inflammatory cells surrounded by a band of prolific vascularisation and fibrosis, and an outer layer of gliosis (Fig. 2A and 3A). Intense perivascular infiltration by inflammatory cells was noted in the cerebral tissue surrounding the abscess (Fig. 2A). Prominent proliferation of connective tissue surrounding the capsular blood vessels was demonstrated among the abscess wall sections stained with Masson's trichrome technique (Fig. 3A). Although necrotic, reactive, fibrous, and encepha- litic zones were demonstrated among the stained section of those abscesses induced by septic embolisation, a deficiency in inflammatory cells was revealed in the medial reactive layer and in the perivascular spaces in the surrounding brain (Figs. $2 B$ and $3 B$ ). The vascular proliferation and the surrounding connective tissue formation within the fibrous layer of these metastatic abscesses were profoundly diminished (Fig. 3B) as compared with that of the traumatic abscess walls (Fig. $3 \mathrm{~A}$ ).

Deep anterior paramedian infarctions or haemorrhage involving the pallidum, caudate nucleus, putamen, internal and external capsules, claustrum, and portions of the cortex were demonstrated on pathological examination of the four monkeys which died within 24 hours of intracarotid embolisation. Profound swelling of the infarcted hemisphere with uncal herniation and secondary midbrain lesions were also present in these monkeys. The pattern of bland infarction occurring in the animals which survived embolisation was similar but spared the globus pallidus and caudate nucleus. Cavitation of the infarcted cerebral tissue without cerebral swelling, uncal herniation or midbrain lesions was observed in the embolised control monkeys.

\section{Discussion}

The production of experimental brain abscesses requires stasis of bacteria in a focus of ischaemic or necrotic brain tissue (Wood et al., 1977a). Such abscesses have been induced in rabbits and dogs by direct instillation of organisms into a focus of
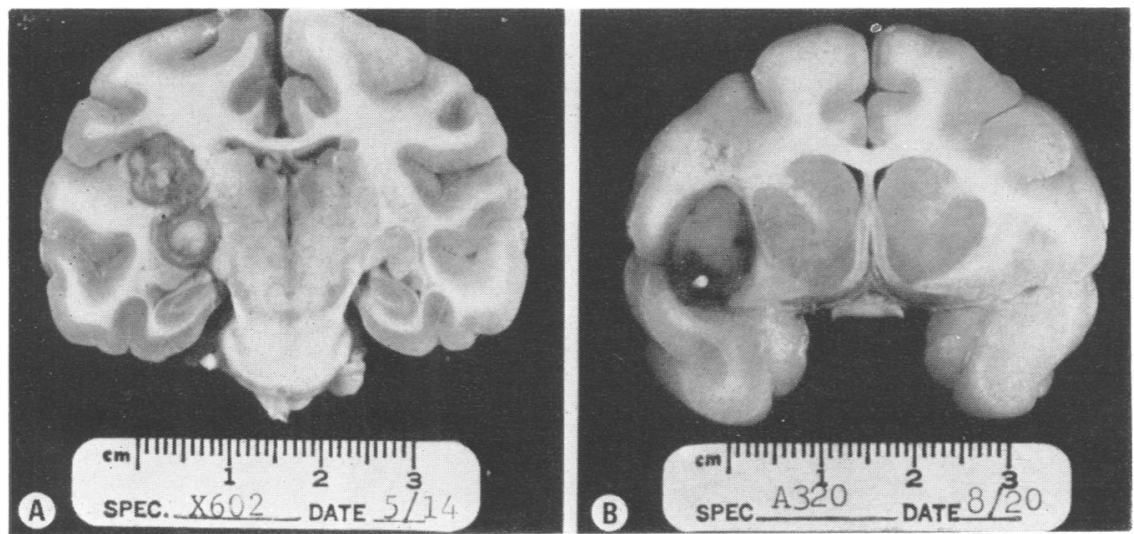

Fig. 1 Coronal brain sections. A: brain abscess induced by intracerebral injection of contaminated nutrient agar exhibits thick capsule wall and abscess budding. Note shift of midline structures away from the abscess. B: brain abscess produced by intracarotid embolisation of septic silicone cylinders demonstrates retarded capsular wall formation. Note cavitation of infarcted tissue dorsal to the abscess and absence of shift of midline structures. 

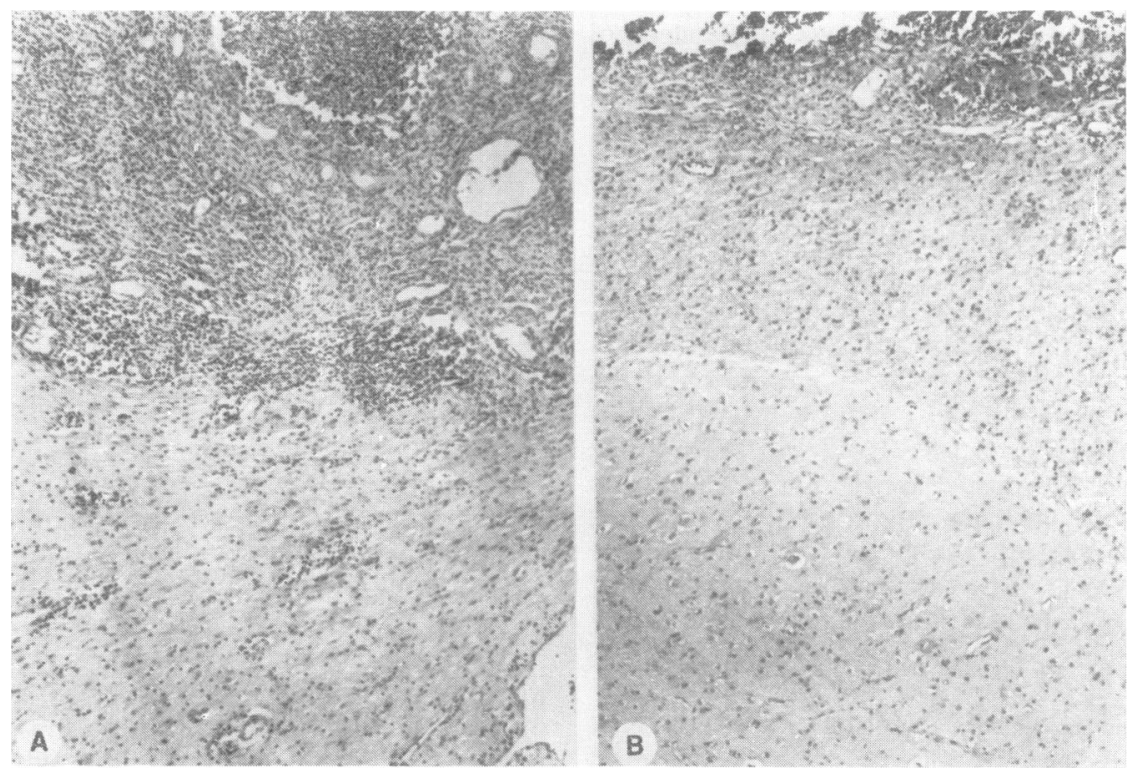

Fig. 2 Photomicrographs of abscess wall during sixth week after operation prepared with luxol fast blue-periodic acid-Schiff stain. A: prominent abscess wall produced by direct intracerebral instillation of contaminated agar demonstrates central necrotic zone, thick medial layer of acute inflammatory cells surrounded by zone of prolific vascularisation and collagen deposition and outer zone of gliosis. Note prominent perivascular infiltration by inflammatory cells in surrounding brain. B: meagre abscess wall induced by septic embolisation demonstrated few inflammatory cells in medial reactive layer and in perivascular spaces in surrounding brain. Note condensation of neuropil and gliotic zone surrounding the abscess.

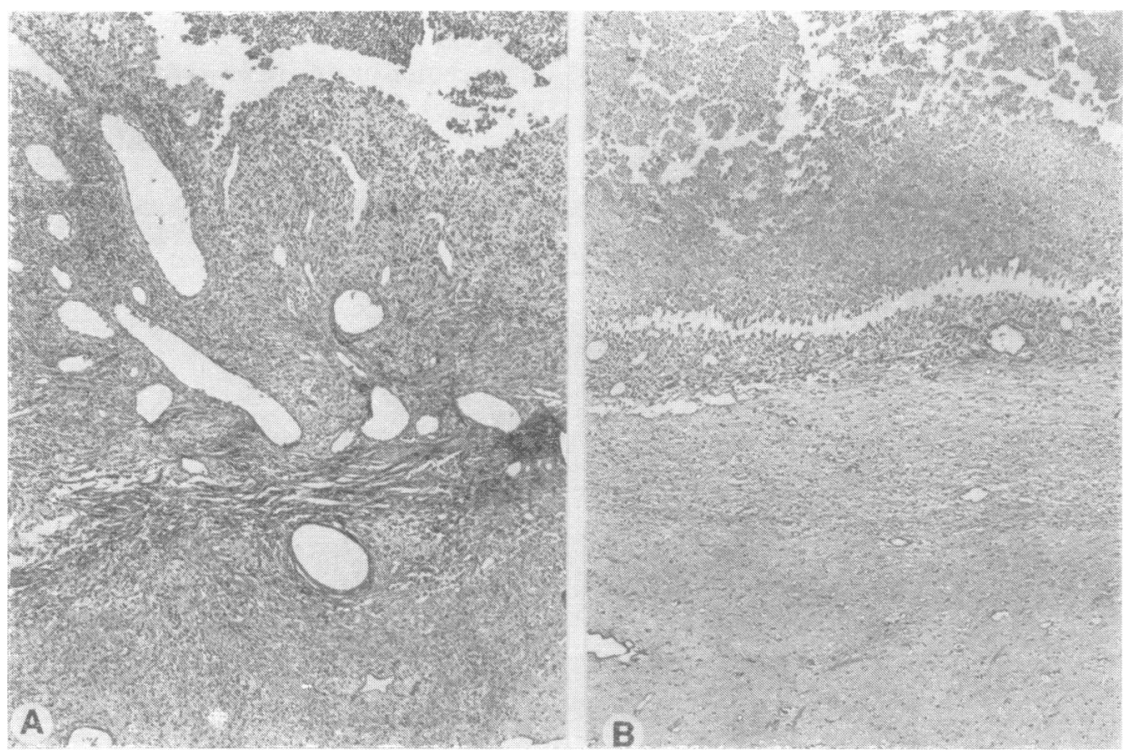

Fig. 3 Photomicrographs of abscess wall during sixth week after operation stained with Masson's trichrome technique. A: prominent capsular wall produced by intracerebral instillation of septic nutrient agar demonstrates collagen deposition surrounding capsular blood vessels. B: meagre capsular wall induced by septic embolisation demonstrated retarded connective tissue formation and vascular proliferation. Note condensation of neuropil surrounding abscess capsule. ( $3 B$ reprinted by permission of Journal of Neurosurgery). 
altered cerebrum (Falconer et al., 1943; Long and Meacham, 1968) and intracarotid injection of contaminated silicone emboli (Molinari, 1972; Molinari et al., 1973). In our primate models, the direct intracerebral inoculation of septic nutrient agar simulated traumatic brain contamination and abscess formation. Our septic intracarotid embolisation induced abscesses which mimicked metastatic brain abscesses.

The degree of encapsulation is relevant to the clinical outcome of the patient (Le Beau et al., 1973). Factors thought to contribute to the development of the capsule include abscess duration, organism characteristics, host resistance, aetiology, and location. The degree of participation of each of these factors is controversial.

Early experimental investigations (Falconer et al., 1943) have indicated that abscess duration is the most important variable in capsular formation. However, retrospective clinical studies have not been conclusive in demonstrating a correlation between the symptom duration and the histological appearance of brain abscesses. Other investigators (Cairnes and Donald, 1935; Kahn, 1969) report that abscesses of long duration usually have thick capsules, whereas acute abscesses are not well encapsulated. On the contrary, later reviewers (Alpers, 1939; Le Beau et al., 1973) note heavy encapsulation in abscesses of short duration, and inadequate capsules in chronic brain abscesses. The degree of encapsulation in our monkeys which received intracerebral injections of contaminated agar was significantly greater than that in our septically embolised animals despite equal ages of abscess in both of these experimental groups (Wood et al., 1978). These data indicate that other factors may make greater contributions to abscess capsule formation in the brain.

Clinical reviewers have concluded that anaerobic or mixed bacterial infections retard encapsulation, whereas aerobic bacteria promote thick capsules (Alpers, 1939). On the other hand, experimental studies suggest no deficiency in encapsulation associated with Gram negative and anaerobic organisms (Falconer et al., 1943). The absence or presence of bacteria in cultures of abscess aspirate did not appear to contribute to the differential encapsulation noted in our two groups of septically inoculated monkeys.

The processes of inflammation and immunity are impeded by glucocorticoid agents. Dexamethasone has been found to retard but not to eliminate the formation of the inflammatory capsule and to preclude the clearance of organisms in experimental brain abscesses (Long and Meacham, 1968; Quartey et al., 1976). Dexamethasone has been noted to reduce inflammatory cell migration (Fruchman, 1964), fibroblastic responses (Regan et al., 1949), and formation of new blood vessels at sites of infection (Ashton and Cook, 1952), but these steroid-induced reductions may vary in different species (Claman, 1972). Unlike rabbits, monkeys are similar to man with respect to their reaction to steroid administration (Claman, 1972). Retarded vascular proliferation, fibrous tissue formation and inflammatory cell infiltration were observed on pathological inspection of abscess walls obtained from our septically embolised monkeys. These findings suggest the possibility that similar inflammatory or immunological deficiencies may exist in devitalised regions of brain after ischaemic infarction.

Previous clinicopathological reviews have noted heavy encapsulation associated with brain abscesses of traumatic origin (Alpers, 1939). Results of those studies drew attention to the participation of adjacent dura mater and pia mater as well as reactive blood vessels in capsular formation. The brain abscesses induced by direct intracerebral inoculation in our animals were surrounded by thick capsules. The microscopic appearance of these experimental abscesses was similar to that described in many displaying the four characteristic zones: the necrotic, reactive, fibrous, and encephalitic layers (Alpers, 1939). No meningeal involvement with the abscess wall was noted in our animals. Proliferating small blood vessels in the reactive zone appeared to be the origin for the abundant capsular collagen observed in our sections stained specifically for connective tissue. Conversely, capsule wall formation and inflammatory responses appeared to be impeded in our septically embolised animals with abscesses. Regional ischaemia secondary to embolic arterial occlusion (Blair and Waltz, 1970) may have compromised capillary proliferation which may cause a retardation of capsular deposition of collagen. Our subsequent study demonstrated a positive relationship between capsular vascularity and collagen formation (Wood et al., 1978).

Capsular formation around a focus of intracerebral infection is not uniform. Fibrosis originating from the reactive vessel walls depends upon the vascularity of the surrounding cerebral tissue. Accordingly, our pathological examination supported the results of previous experimental studies (Falconer et al., 1943) by demonstrating more prominent deposition of collagen in those portions of the abscess adjacent to grey matter as compared with those surrounded by the relatively less vascular white matter. This differential rate of encapsulation in grey and white matter may account 
for the tendency of cerebral abscesses to extend toward the ventricles (Falconer et al., 1943). The location of the abscesses produced in our animals was similar; thus the normal variability in brain vascularity did not contribute significantly to the pathological differences in capsule formation demonstrated in our two experimental groups.

In our recent investigations of these abscess models, serial cerebral angiography demonstrated prominent vascular blushes only in the animals which developed thick encapsulation after intracerebral instillation of contaminated agar, but not in similarly sized metastic abscesses (Wood et al., 1978). That study suggests that capsular blushes are secondary to vascular proliferation within the capsule and not to compression of the surrounding brain.

All animals killed after intracerebral inoculation with contaminated agar demonstrated significant displacement of the midline structures without uncal herniation. This shift of the midline structures appears to be produced by the mass effect of the abscess, because such displacements were not present in the control animals which received sterile agar instillations.

On the other hand, the embolised animals which developed abscesses did not have midline displacements when killed. Apparently, cavitation of the infarcted brain surrounding these metastatic abscesses may have compensated spatially for the mass effect of the growing abscess. Serial angiographic studies of these abscess models demonstrated progressive midline shifts in the septically injected monkeys but only transient early midline displacements in the embolised monkeys (Wood et al., 1978). This resolution of the midline shift in embolised monkeys by the fourth week after operation is in agreement with the observation of resolution of postinfarction oedema in man (Masdeu et al., 1977).

In conclusion, the degree of encapsulation contributes profoundly to patient mortality; thus methods to enhance the proliferation of the capsule wall would have therapeutic significance. These two primate models may be valuable for the study of abscess encapsulation. Additional information on abscess-induced increases in intracranial pressure and alterations in antibiotic pharmacokinetics in the cerebrospinal fluid may be gained by implanting our previously described cerebrospinal fluid reservoir system in these primate abscess models (Wood et al., 1977b, c; Poplack et al., 1977).

\section{References}

Alpers, B. J. (1939). Abscess of the brain: relations of the histologic to the clinical features. Archives of Otolaryngology, 29, 200-222.

Ashton, N., and Cook, C. (1952). In vivo observation of the effect of cortisone upon the blood vessels in rabbit ear chamber. British Journal of Experimental Pathology, 33, 445-450.

Bhatia, R., Tandon, P. N., Banerji, A. K., and Prakash, B. (1976). Brain abscess and congenital heart disease. Acta Neurochirurgica, 33, 233-239.

Blair, R. D. G., and Waltz, A. G. (1970). Regional cerebral blood flow during acute ischemia: correlation of autoradiologic measurements with observations of cortical microcirculation. Neurology (Minneapolis), 20, 802-808.

Cairnes, H., and Donald, D. (1935). The diagnosis and treatment of abscess of brain. Journal of Laryngology and Otology, 50, 73-80.

Carey, M. E., Chou, S. N., and French, L. A. (1972). Experience with brain abscesses. Journal of Neurosurgery, 36, 1-9.

Claman, H. N. (1972). Corticosteroids and lymphoid cells. New England Journal of Medicine, 287, 388397.

Eberhard, S. J. (1969). Diagnosis of brain abscess in infants and children, a retrospective study of 26 cases. North Carolina Medical Journal, 30, 301-313.

Falconer, M. A., McFarlin, A. M., and Russell, D. S. (1943). Experimental brain abscess in the rabbit. British Journal of Surgery, 30, 245-260.

Fruchman, G. J. (1964). Extravascular mobilisation of neutrophils. Annals of New York Academy of Science, 113, 968-1002.

Garfield, J. (1969). Management of supratentorial intracranial abscess: a review of 200 cases. British Medical Journal, 2, 7-11.

Hassler, O., and Forsgren, A. (1964). Experimental. abscesses in brain and subcutis: a microangiographic study in the rabbit. Acta Pathologica et Microbiologica Scandinavica, 62, 59-67.

Hoffman, H. J., Hendrick, E. B., and Hiscox, J. L. (1970). Cerebral abscesses in early infancy. Journal of Neurosurgery, 33, 172-177.

Joubert, M. J., and Stephanov, S. (1977). Computerized tomography and surgical treatment of intracranial suppuration; report of 30 consecutive unselected cases of brain abscess and subdural empyema. Jour nal of Neurosurgery, 47, 73-78.

Kahn, E. A. (1969). Brain abscess. In Correlative Neurosurgery, pp. 253-265. Edited by E. A. Kahn, E. C. Crosby, R. C. Schneider, and J. A. Taren. Charles C. Thomas: Springfield, Illinois.

Kaufman, D. M., and Leeds, N. E. (1977). Computed tomography (CT) in the diagnosis of intracranial abscesses: brain abscess, subdural empyema, and epidural empyema. Neurology (Minneapolis), 27, 1069-1073.

Laurent, J. P., Molinari, G. F., and Mosely, J. I. (1975). Clinicopathologic validation of a primate stroke model. Surgical Neurology, 4, 449-455.

Laurent, J. P., Molinari, G. F., and Oakley, J. R. (1976). Primate model of cerebral hematoma. Jour 
nal of Neuropathology and Experimental Neurology, 35, 560-568.

Le Beau, J., Creissard, P., Harispe, L., and Redondo, A. (1973). Surgical treatment of brain abscess and subdural empyema. Journal of Neurosurgery, 38, 198-203.

Long, W. D., and Meacham, W. F. (1968). Experimental model for producing brain abscess in dogs with evaluation of the effect of dexamethasone and antibiotic therapy on pathogenesis of intracerebral abscess. Surgical Forum, 19, 437-438.

Masdeu, J. C., Azar-kia, B., and Rubino, F. A. (1977). Evaluation of recent cerebral infarction by computerized tomography. Archives of Neurology (Chicago), 34, 417-421.

Molinari, G. F. (1972). Septic cerebral embolism. Stroke, 3, 117-122.

Molinari, G. F., Smith, L., Goldstein, M. N., and Satran, R. (1973). Pathogenesis of cerebral mycotic aneurysms. Neurology (Minneapolis), 23, 325-332.

New, P. F. J., David, K. R., and Ballantine, H. T. (1976). Computed tomography in cerebral abscess. Radiology, 121, 641-646.

Poplack, D. G., Bleyer, W. A., Wood, J. H., Kostolich, M., Savitch, J. L., and Ommaya, A. G. (1977). A primate model for study of methotrexate pharmacokinetics in the central nervous system. Cancer Research, 37, 1982-1985.

Quartey, G. R. C., Johnston, J. A., and Rozdilsky, B. (1976). Decadron in the treatment of cerebral abscess, an experimental study. Journal of Neurosurgery, 45, 301-310.

Regan, C., Howes, E. L., and Plotz, C. M. (1949). Effect of cortisone on production of granulation tissue in the rabbit. Proceedings of Society of Experimental Biological Medicine, 72, 718-721.

Shaw, M. D. M., and Russell, J. A. (1975). Cerebellar abscess: a review of 47 cases. Journal of Neurology, Neurosurgery, and Psychiatry, 38, 429-435.
Shaw, M. D. M., and Russell, J. A. (1977). Value of computed tomography in the diagnosis of intracranial abscess. Journal of Neurology, Neurosurgery, and Psychiatry, 40, 214-220.

Van Alphen, H. A. M., and Dreissen, J. J. R. (1976). Brain abscess and subdural empyema: factors influencing mortality and the results of various surgical techniques. Journal of Neurology, Neurosurgery, and Psychiatry, 39, 481-490.

Wood, J. H., Doppman, J. L., Lightfoote, W. E., Girton, M., and Ommaya, A. K. (1978). Role of vascular proliferation on angiographic appearance and encapsulation of experimental traumatic and metastatic brain abscesses. Journal of Neurosurgery, 48, 264-273.

Wood, J. H., Parver, M., Doppman, J. L., and Ommaya, A. K. (1977a). Experimental intraoperative localization of retained intracerebral bone fragments using transdural ultrasound. Journal of Neurosurgery, 46, 65-71.

Wood, J. H., Poplack, D. G., Bleyer, W. A., and Ommaya, A. K. (1977b). Primate model for longterm study of intraventricularly or intrathecally administered drugs and intracranial pressure. Science, 195, 499-501.

Wood, J. H., Poplack, D. G., Flor, W. J., Gunby, E. N., and Ommaya, A. K. (1977c). Chronic ventricular cerebrospinal fluid sampling, drug injections and pressure monitoring using subcutaneous reservoirs in monkeys. Neurosurgery, 1, 132-135.

Zimmerman, R. A., Bilaniuk, L. T., Shipkin, P. M., Gilden, D. H., and Murtagh, F. (1977). Evaluation of cerebral abscess: correlation of clinical features with computed tomography. Neurology (Minneapolis), 27, 14-19.

Zimmerman, R. A., Patel, S., and Bilaniuk, L. T. (1976). Demonstration of purulent bacterial intracranial infections by computed tomography. American Journal of Roentgenology, 127, 155-165. 